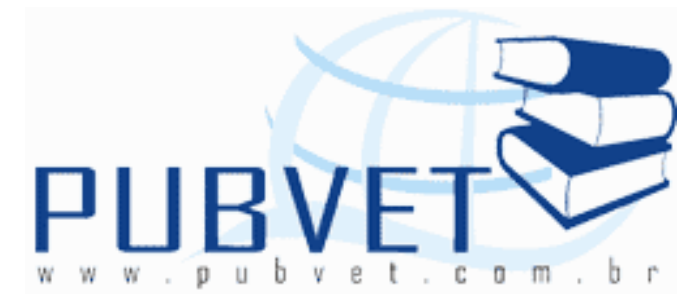

PUBVET, Publicações em Medicina Veterinária e Zootecnia.

\title{
Atendimento ambulatorial de quati-de-cauda-anelada (Nasua nasua) Relato de caso
}

Carolina Pereira Soares ${ }^{1}$, Bárbara Teresa Tavares Martins ${ }^{1}$, Adairton da Silva Lima $^{2}$, Gabriel Domingos Carvalho ${ }^{3}$, Cláudia Sampaio ${ }^{4}$, Moacir Carretta Junior ${ }^{4}$

${ }^{1}$ Graduanda do Curso de Medicina Veterinária da Faculdade de Ciências Biológicas e da Saúde (FACISA/UNIVIÇOSA), Viçosa-MG.

${ }^{2}$ Médico Veterinário - Laboratório IPEVE, Belo Horizonte.

${ }^{3}$ Professor do curso de Med. Veterinária do Instituto Federal Norte de Minas Gerais - IFNMG Campus Salinas, Salinas-MG.

${ }^{4}$ Professor(a) do curso de Med. Veterinária FACISA/UNIVIÇOSA, Viçosa-MG.

Autor para correspondência: Gabriel Domingos Carvalho

E-mail: gabriel.carvalho@ifnmg.edu.br

Endereço: Fazenda Varginha, Km 02, Rod. Salinas/Taiobeiras. 39.560-000. Salinas, MG. Tel./Fax. +55 3838417000.

\section{Resumo}

Quatis são animais gregários, que podem viver em grupos de mais de trinta indivíduos e possuem alta adaptabilidade. Por serem animais sinantrópicos, sua presença próxima aos humanos e aos animais domésticos oferece riscos à saúde pública, pois são transmissores de algumas zoonoses. Este trabalho relata o resgate e o atendimento clínico de um quati-de-cauda-anelada (Nasua nasua), que apresentava um quadro severo de prostração e infestação por 
miíases e carrapatos. O animal foi mantido em cativeiro para realização do tratamento. Após a melhora clínica substancial, estando ativo e com sinais de recuperação total o animal foi posto em liberdade no mesmo local onde foi encontrado.

Palavras-chave: quati, Nasua nasua, animais sinantrópicos.

\title{
Ambulatory care of a ring-tailed coati (Nasua nasua) - Case report
}

\begin{abstract}
Coatis are gregarious animals that can live in groups of more than thirty individuals and have high adaptability. Because they are synanthropic animals, their presence next to humans and domestic animals offers public health risks because they are transmitters of some zoonoses. This paper reports the rescue and clinical care of a ring-tailed coati (Nasua nasua), who presented a case of severe prostration and infestation by myiasis and ticks. The animal was kept in captivity for completion of treatment. After the substantial clinical improvement, being active and with signs of complete recovery the animal was released at the same place where he was found.
\end{abstract}

Keywords: coati, Nasua nasua, synanthropic animals.

\section{INTRODUÇÃO}

Os quatis pertencem ao filo Chordata, classe Mammalia, ordem Carnivora e família Procyonydae, gênero Nasua (VERÍSSIMO et al., 2008). São reconhecidas duas espécies do gênero, Nasua narica (quati-de-nariz-branco) e Nasua nasua (quati da América do Sul, quati-do-nariz-marrom ou quati-decauda-anelada), ambas restritas ao continente Americano (BONATTI, 2006).

O quati é identificado pelo formato do corpo e pelo focinho longo que se destaca diante dos olhos e orelhas pequenas (FERRI et al., 2008). O corpo dos adultos mede de 40 a 65 centímetros de comprimento (RIBEIRO et al., 2011), podendo chegar a ter $30,5 \mathrm{~cm}$ de altura (GREGORES, 2006), seu peso pode variar de 2,7 a 10 quilogramas (RIBEIRO et al., 2011). São animais de porte 
médio, pernas curtas e pelagem densa. São plantígrados, possuindo cinco dedos bem desenvolvidos em todas as patas e as garras não são retráteis. As mãos móveis permitem habilidade para cavar e Ihes conferem o título de bons escaladores (CUBAS et al., 2007), por isso criam seus filhotes em ninhos arbóreos (CAMPOS, 2009).

São animais onívoros, capazes de adquirir os nutrientes essenciais por meio de uma grande variedade alimentar na natureza (CUBAS et al., 2007), que inclui, frutas, insetos, ovos e pequenos vertebrados (FERRI et al, 2008). Também possuem hábito forrageio e o focinho flexível é usado para revolver qualquer material encontrado no chão (WHITEMAN, 2007). Possuem um papel muito importante por serem espécies dispersoras de sementes, por defecá-las intactas, contribuindo assim para a conservação das vegetações (BARCELOS; HEMETRIO; CARVALHO, 2011). Possuem os dentes molares largos e bem adaptados ao esmagamento dos alimentos (CAMPOS, 2009), são fortes e afiados podendo causar sérias lesões em outros animais (PIERI et al., 2011).

Os quatis são animais gregários, podendo viver em grupos de mais de 30 indivíduos, variando em decorrência de disponibilidade de recursos. Os machos possuem porte mais avantajado, sendo geralmente solitários fora da época reprodutiva (BARROS e FRENEDOZO, 2010) e possuem hábito noturno, enquanto as fêmeas e os filhotes são ativos durante o dia (MATTGE et.al.; 2002).

A espécie possui alta adaptabilidade gerando aumento de sua população, tanto que mesmo na periferia de cidades é possível observar bandos de quatis como animais sinantrópicos, sobrevivendo de restos alimentares, acarretando conflitos com humanos e outras espécies, além do aumento do risco de transmissão de zoonoses (CAMPOS, 2009), com a participação no ciclo de algumas doenças como a leptospirose e tripanossomíase (RIBEIRO et al., 2011).

A grande densidade populacional, que os quatis apresentam, devido a restrição de habitat (WHITEMAN, 2007), faz com que em muitos locais ele seja a espécie de carnívoro mais abundante (ROCHA, 2006). Estas podem gerar um 
impacto negativo levando ao declínio de algumas populações da própria fauna silvestre ou mesmo acometer animais domésticos (SILVA, 2004). A perda do seu habitat, causada pela invasão humana ao ambiente de floresta, ocasiona mudanças claramente percebidas quando estes animais se aproximam de residências humanas a procura de alimento (WHITEMAN, 2007). Sassi et al. (2009) relatam o quati como uma das espécies de mamíferos vítimas do atropelamento em rodovias de minas gerais.

Este estudo de caso tem por objetivo relatar o atendimento de um quatide-cauda-anelada (Nasua nasua) encontrado com miíases subcutâneas em área urbana, necessitando de cuidados médico-veterinário.

\section{RELATO DE CASO}

Um exemplar adulto, macho de quati-de-cauda-anelada (Nasua nasua), com massa corpórea de $4 \mathrm{~kg}$, apresentava um quadro de prostração, alta infestação por carrapatos e miíase cutânea no pescoço e toda região abdominal ventral, principalmente na região prepucial (Figura 1) foi encaminhado para atedimento médico veterinário. $O$ animal foi encontrado em um lote bastante arborizado, em área urbana do município de Rio Doce, Zona da Mata do Estado de Minas Gerais, Brasil. Moradores do local relataram que quatis são frequentemente observados nas redondezas, em busca de alimentação.

O animal foi recolhido por populares e encaminhado ao atendimento médico-veterinário, sob consentimento da Polícia Militar Ambiental. O Centro de Triagem de Animais Silvestres do Departamento de Veterinária da Universidade Federal de Viçosa (CETAS/DVT/UFV) também foi contactado para se obter orientações.

O animal foi contido e realizou-se tricotomia e anti-sepsia de toda região cervical e abdominal. Fez a retirada manual dos ectoparasitas, sendo identificados carrapatos do gênero Amblyomma. Fez também a higienização das feridas cutâneas e retirada de larvas de dípteros pertencente à família Calliphoridae, característicos do gênero Cochliomyia. 
Frente o quadro apresentado pelo quati, suspeita-se que ele possa ter se envolvido em alguma luta por disputa territorial com outro macho, ou que ele possa ter sido vítima de algum ataque por outros animais, como cães.

Foram administrados parenteralmente: $0,5 \mathrm{~mL}$ de penicilina $(0,5 \mathrm{~mL} / 5 \mathrm{~kg})$ por via intramuscular; $0,1 \mathrm{~mL}$ de doramectina $(1 \mathrm{~mL} / 50 \mathrm{~kg})$ por via subcutânea e $0,25 \mathrm{~mL}$ dipropionato de imidocarb $(12 \mathrm{~g} / 100 \mathrm{~mL})$ subcutâneo. O animal foi mantido em internação ambulatorial para observação.

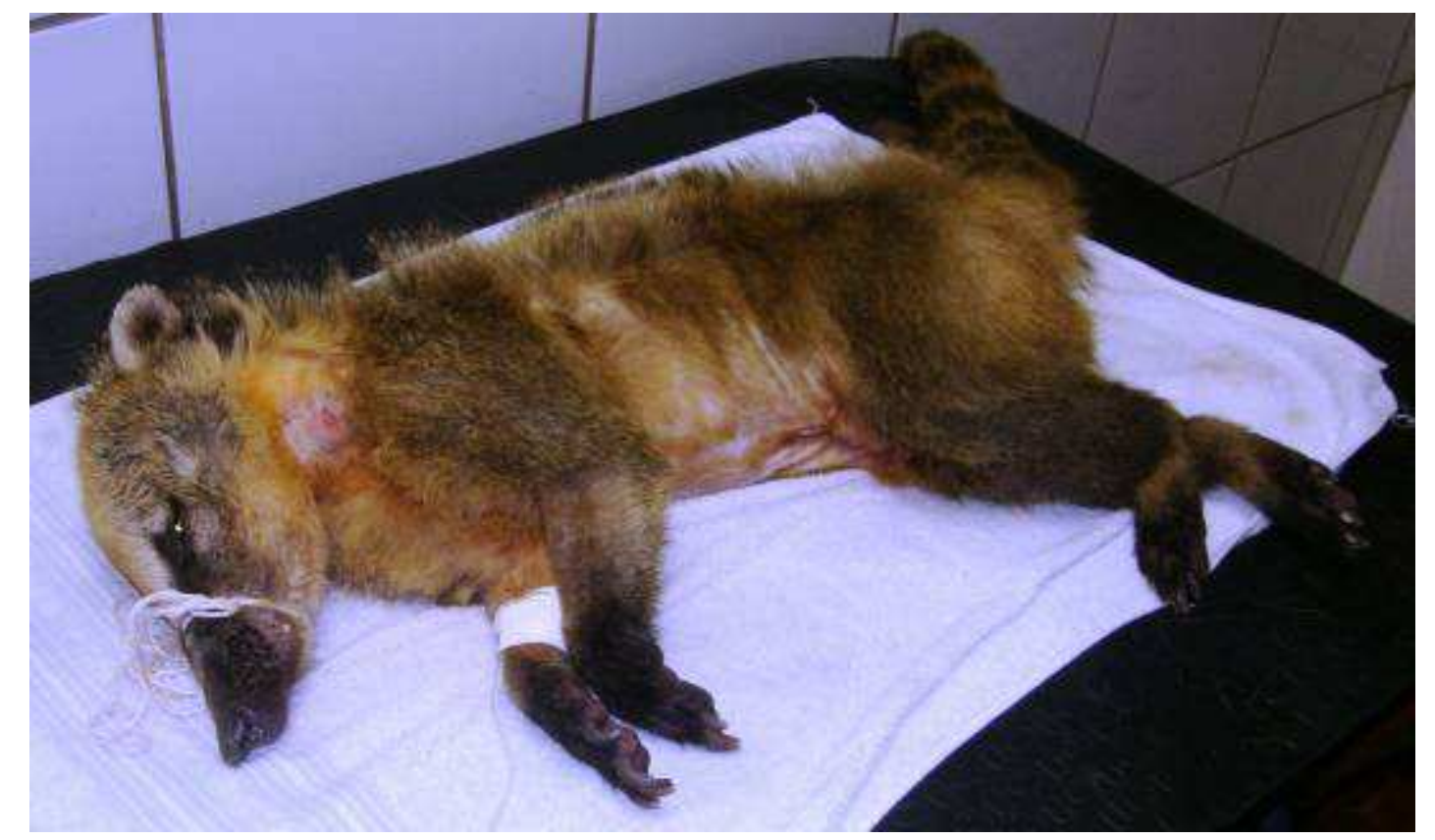

Figura 1. Quati-de-cauda-anelada (Nasua nasua) apresentando quadro de prostração, alta infestação por carrapatos e miíase cutânea, encaminhado para atendimento ambulatorial.

Foi realizado hemograma do animal (Tabela 1 ), sendo que ele apresentava um quadro de anemia macrocítica hipocrômica, leucopenia com neutropenia e trombocitopenia, sugestivo de um quadro de infecção crônica.

No dia seguinte o animal não apresentava nenhuma reação clínica e deuse continuidade ao tratamento. Ele foi sedado com uma associação de $0,5 \mathrm{~mL}$ de cetamina $(5 \mathrm{mg} / \mathrm{kg})$ com $0,2 \mathrm{~mL}$ xilazina $(1 \mathrm{mg} / \mathrm{Kg})$, administrados 10 minutos após ter recebido $0,1 \mathrm{~mL}$ de atropina $(0,02 \mathrm{mg} / \mathrm{Kg})$ por via intra 
muscular. Também foi usado um spray de permetrina e 1 comprimido de vermífugo (nitenpiram 11,4mg), juntamente com 0,5 $\mathrm{mL}$ de dexametasona (1 $1 \mathrm{mg})$ e $1 \mathrm{~mL}$ de enrofloxacina $(1 \mathrm{~mL} / 5 \mathrm{~kg})$, vias intra muscular. $O$ animal apresentava-se desidratado e sem se alimentar, por isso recebeu fluidoterapia parenteral $(500 \mathrm{~mL}$ de solução de ringer lactado e $500 \mathrm{~mL}$ de soro fisiológico glicosado $5 \%$ ).

Tabela 1. Resultados do hemograma realizado no quati (Nasua nasua) no durante $o$ atendimento ambulatorial.

\begin{tabular}{cc}
\hline Resultado & $\begin{array}{c}\text { Valores de Referência } \\
\text { (FOWLER e MILLER, 2003) }\end{array}$ \\
\hline
\end{tabular}

\section{Série Vermelha}

Hemácias

Hemoglobina

Hematócrito

V.G.M.

H.G.M.

C.H.G.M.

$$
4,99 \mathrm{milh} / \mathrm{mm} 3
$$$$
10,10 \mathrm{~g} / \mathrm{dl}
$$$$
33,80 \%
$$$$
67,74 \text { u3 }
$$$$
20,24 \mathrm{pg}
$$$$
29,88 \%
$$

\section{Série Branca}

Leucócitos Global

Blastonetes

Promielócitos

Mielócitos

Metamielócitos

Bastonetes

Segmentados

Eosinófilos

Basófilos

Linfócitos

Monócitos
6.200 lec.

$$
0
$$$$
0
$$

0

0

248

3.844

62

0

1.922

124
$6,97 \pm 0,93$

$12,0 \pm 1,5$

$37,1 \pm 5,0$

$53,3 \pm 7,1$

$17,2 \pm 2,4$

$32,4 \pm 2,9$

$$
10,6 \pm 4,32
$$

$7,95 \pm 3,99$

$0,39 \pm 0,27$

$0,15 \pm 0,14$

$1,92 \pm 1,35$

$0,38 \pm 0,31$ 
Nos terceiro e quarto dias ele apresentou melhora clínica gradativa, porém ainda recusava a alimentação. Repetiu-se a medicação e a fluidoterapia administradas anteriormente.

A partir do quinto dia o animal já apresentava apetite voraz (tinha preferência por ovos de galinha e frutas como banana e mamão) e sua melhora era visivelmente positiva. Continuou-se a administração de dexametazona (a dose foi diminuída gradativamente) e enrofloxacina até completar-se sete dias de tratamento.

O animal permaneceu em observação até completar o décimo quinto dia, quando foi realizada outra dose de dipropionato de imidocarb subcutâneo. Após terminado o tratamento e o paciente ter apresentado uma melhora clínica substancial, ele foi levado ao mesmo local onde foi encontrado e foi posto em liberdade, estando ativo e com sinais de recuperação total.

\section{CONCLUSÃO}

A redução de habitats que advém de ações como exploração de madereira, expansão agrícola, industrial e urbana, em conjunto com a grande adaptabilidade dos quatis faz com que essa espécie se aproxime cada vez mais dos humanos e animais domésticos, acarretando problemas diversos como transmissão de doenças zoonóticas, além da ocorrência de atropelamentos de quatis. A recuperação de habitats e a preservação ambiental é uma via que permite que esses animais vivam de forma natural e sem trazer riscos à saúde pública.

\section{REFERÊNCIAS BIBLIOGRÁFICAS}

BARCELOS, D.C.; HEMETRIO, N.S.; CARVALHO, A.F.M.M. Avaliação da Relação Visitantequati como ferramenta para criação de estratégias de Educação Ambiental no Parque das Mangabeiras, Belo Horizonte, MG. X Congresso de Ecologia do Brasil, São LourençoMG, 2011. p. 1.

BARROS, D.; FRENEDOZO, R.C. Uso do habitat, estrutura social e aspectos básicos da etologia de um grupo de quatis (Nasua nasua Linnaeus, 1766) (Carnívora: Procyonidae) em uma área de Mata Atlântica, São Paulo, Brasil. Biotemas, 2010. 
BONATTI, J. Uso e Seleção de Habitat, Atividade Diária e Comportamento de Nasua nasua (Linnaeus, 1766) (Carnivora; Procyonidae) na Ilha do Campeche, Florianópolis, Santa Catarina. Dissertação de Mestrado - Universidade Federal do Rio Grande do Sul, Porto Alegre, 2006. p. 21-137.

CAMPOS, P.K.A. Avaliação Morfofuncional do Testículo de Quatis (Nasua nasua Linnaeus, 1766) Adultos. Dissertação de Mestrado - Universidade Federal de Viçosa, Viçosa, 2009. p. 1- 5.

CUBAS, Z.S.; SILVA, J.C.R.; CATÃO-DIAS, J.L. Tratado de Animais Selvagens. São Paulo: Roca, 2007. p. 571-583.

FERRI, R.C. et al. Eletrocardiografia em quatis (Nasua nasua - Linnaeus, 1766) mantidos em cativeiro e contidos quimicamente com quetamina e xilazina. Revista Clínica Veterinária, n. 74, 2008. p. 68.

GREGORES, G.B. Topografia vértebra-medular e Anestesia Espinhal em Quati (Nasua nasua). Dissertação de Mestrado - Faculdade de Medicina Veterinária e Zootecnia da Universidade de São Paulo, São Paulo, 2006. p. 18-63.

MATTGE, G.I.; KESTRING, D.; KLEIN, J.; NASCIMENTO, A.F. O estudo da formação de grandes bandos de quatis (Nasua nasua Linnaeu, 1766) no espaço semi-fechado do Parque Ecológico Diva Paim Barth no município de Toledo-PR. XI Encontro Anual de Iniciação Científica, Maringá-PR, 2002.

PIERI N.C.G.; MANÇANARES, C.A.F.; BERTASSOLI, B.; LIMA, J.M.N.; THOMAZ, J. M.; CARVALHO,A.F . CLASSIFICAÇÃO MORFOFUNCIONAL DOS DENTES DE QUATI, NASUA nasua. Pesquisa Veterinária Brasileira, 2011. p 447.

RIBEIRO, R.G. et al. Padronização do Exame Ultrassonográfico do Abdomen de Quatis (Nasua nasua).Goiânia, Goiás, 2011.

ROCHA, F.L. Áreas de Uso e Seleção de Habitats de três Espécies de Carnívoros de Médio Porte na Fazenha Nhumirim e Arredores, Pantanal de Nhecolândia, MS. Dissertação apresentada ao programa de Pós-graduação em Ecologia e Conservação da Universidade Federal de Mato Grosso do Sul, Corumbá, 2006.

SÁSSI, C.M.; NASCIMENTO, A.A.; MIRANDA, R.F.P. \& CARVALHO, G.D. 2009. Impacto do atropelamento de animais silvestres em trecho da BR482, Minas Gerais-BR. In II Congresso Internacional de Medicina da Conservação. Universidade Federal Rural de Pernambuco, Recife. p.91.

SILVA, R.A.A.S.; LIMA, E.S.S. Alterações Hematológicas e Bioquímicas em Quatis (Nasua nasua) naturalmente infectados por Trypanosoma evansi no Pantanal, MS. Circular Técnica 55 EMBRAPA, Corumbá - MS, 2004.

VERÍSSIMO, S. et al. Comportamento Animal do Quati (Nasua nasua) no Zoológico do Parque do Sabiá de Uberlândia - MG e Propostas de Enriquecimento do Cativeiro. In: XX Semana Científica de Medicina Veterinária de Uberlândia e V Mostra de Pós-Graduação em Ciências Veterinárias da Faculdade de Medicina Veterinária - UFU, 2008 p. 84-85.

WHITEMAN, C.W. Conservação de carnívoros e a interface homem-fauna domésticafauna silvestre numa área fragmentada da Amazônia oriental brasileira. Tese para Título de Doutor em Ecologia Aplicada. Piracicaba, 2007. p. 52-54. 